\title{
ON GARSIA'S CRITERION FOR UNIFORM CONVERGENCE OF FOURIER SERIES
}

\author{
CHARLES OEHRING
}

(Received 11 November 1989; revised 11 April 1990)

Communicated by W. Moran

\begin{abstract}
Garsia's discovery that functions in the periodic Besov space $\Lambda\left(p^{-1}, p, 1\right)$, with $1<p<\infty$, have uniformly convergent Fourier series prompted him, and others, to seek a proof based on one of the standard convergence tests. We show that Lebesgue's test is adequate, whereas Garsia's criterion is independent of other classical critiera (for example, that of Dini-Lipschitz). The method of proof also produces a sharp estimate for the rate of uniform convergence for functions in $\Lambda\left(p^{-1}, p, 1\right)$. Further, it leads to a very simple proof of the embedding theorem for these spaces, which extends (though less simply) to $\Lambda(\alpha, p, q)$.
\end{abstract}

1980 Mathematics subject classification (Amer. Math. Soc.) (1985 Revision): 42 A 20.

\section{Introduction}

In an article [8] striking for novel derivations of facts about Fourier series Garsia proved an inequality that implies the uniform convergence of the Fourier series of functions that belong to the Besov space $\Lambda\left(p^{-1}, p, 1\right)$; that is, of each $f$ whose $L^{p}$ modulus of continuity satisfies

$$
\int_{0}^{1} \omega(h ; p ; f) h^{-1-1 / p} d h<\infty,(1 \leq p<\infty) .
$$

He also offered proof along more customary lines but later, in [9], reflected whether a proof of uniform convergence could be based directly upon one of the well known convergence criteria for Fourier series.

Recently, this invitation was accepted by Fournier and Self [7]; however,

(C) 1991 Australian Mathematical Society 0263-6115/91 \$A2.00+0.00 
the criterion for uniform convergence that these authors bring to bear is questionably "well-known" (hence all the more valuable). In Section 2 we use simple, rudimentary (with one exception) facts to deduce Garsia's theorem from the convergence test of Lebesgue.

Fournier and Self extend their techniques to discuss allied questions and a number of connections with other work. For example, they point out that an obscure 1907 criterion of Hobson $\left(\int_{0}^{1} \omega(h ; f) h^{-1} d h<\infty\right)$ is a limiting version of Garsia's criterion. Fournier and Self remark upon the presumptive independence of these criteria but forgo its verification. Like Garsia's theorem the Dini-Lipschitz theorem (see Section 4) can be deduced [11, p. 45] from Lebesgue's test; and Hobson's theorem is a ready consequence of the Dini-Lipschitz theorem. In Section 4 we verify that Garsia's theorem does not contain Hobson's theorem, nor is it implied by the Dini-Lipschitz theorem.

In the spirit of Garsia's query it is appropriate that our initial proof, ir Section 2, be as elementary as feasible. In Section 5 we augment the proof with Riesz's theorem to obtain a natural, sharp estimate for the rate of uni. form convergence in terms of the $p$-modulus of continuity. A seemingl! novel corollary of this estimate implies, in particular, that if $f^{\prime}$ belongs $t$, $\operatorname{Lip}(\alpha, p)$, with $1<p<\infty$, then $f(x)-S_{n}(x)=O\left(1 / n^{1+\alpha-1 / p}\right)$, uniforml: on $[0,2 \pi]$.

If $f$ satisfies Garsia's criterion for some value of $p<\infty$, it satisfies th criterion for all larger values of $p$. This fact, essential for a satisfactor. understanding of Garsia's theorem, is proved in [7] (see also [21, p. 16i]). Is Section 6 we prove a more general result, based upon the method developer in Section 5.

\section{Garsia's Theorem}

Suppose that $1 \leq p \leq \infty, h>0, f$ is $2 \pi$-periodic (tacitly assumer henceforth), and $f \in L^{p}[0,2 \pi]$. We write

$$
\begin{gathered}
\Omega(h ; p ; f) \doteq\|f(\cdot+h)-f(\cdot)\|_{L^{p}} \text { if } p \neq \infty, \\
\Omega(h ; \infty ; f) \doteq \sup |f(\cdot+h)-f(\cdot)|
\end{gathered}
$$

and $\omega(h ; p ; f) \doteq \sup \left\{\Omega\left(h^{\prime} ; p ; f\right): 0<h^{\prime} \leq h\right\}$. For $f \in L^{p} \quad(f \in C$ it $p=\infty)$ we let $E(n ; p ; f) \doteq \inf \left\{\left\|f-T_{n}\right\|_{p}: T_{n}\right.$ is a trigonometric polynomia of degree $\leq n\}$. Weierstrass's theorem asserts that $E(n ; \infty ; f) \downarrow 0$ for ever) continuous $f$; whence $E(n ; p ; f) \downarrow 0$ for $f \in L^{p}(1 \leq p<\infty)$. Jackson': "first" theorem [5, p. 97] guarantees that $E(n ; p ; f)=O\left(\omega\left(n^{-1} ; p ; f\right)\right)$ it $1 \leq p \leq \infty$. Thus, by comparison with a series and condensation $[13$, p. 120 . 
(see also the proof of Theorem 5.1),

$$
\int_{0}^{1} \omega(h ; p ; f) h^{-1-1 / p} d h<\infty
$$

implies that $\sum_{0}^{\infty} 2^{\nu / p} E\left(2^{\nu} ; p ; f\right)<\infty$. We observe also that finiteness of the integral requires, for $h$ small, that

$$
\varepsilon>\int_{h}^{2 h} \omega(\lambda ; p ; f) \lambda^{-(1+1 / p)} d \lambda \geq h \omega(h ; p ; f)(2 h)^{-(1+1 / p)} ;
$$

that is, that $\omega(h ; p ; f)=o\left(h^{1 / p}\right)$. In particular, if $p=1, f$ must be constant $[22$, p. 45$]$.

REMARK 2.1. We shall eventually require the inequality

$$
\|T\|_{r} \leq A_{p, r} n^{1 / p-1 / r}\|T\|_{p},
$$

where $T$ is a trigonometric polynomial of degree $\leq n$ and $1 \leq p \leq r \leq \infty$. The proof $[22$, p. 154] is rather sophisticated. To prove Garsia's Theorem we use the case $r=\infty$, which Garsia observed in [8] is a consequence of Bernstein's inequality. In fact, the idea behind the proof of Bernstein's inequality in $[22$, p. 118$]$, leads directly to $\|T\|_{\infty} \leq 3 n^{1 / p}\|T\|_{p}$. See the Appendix.

Turning to the proof of Garsia's theorem we suppose that $f \in L^{p} \quad(1 \leq$ $p<\infty)$ and consider a sequence of polynomials $T_{n} \equiv T(n)$ for which (degree of $\left.T_{n}\right) \leq n$ and $\left\|f-T_{n}\right\|_{p} \leq 2 E(n ; p ; f)$. Since $\left\|f-T\left(2^{m+1}\right)\right\|_{p} \rightarrow 0$ we have the representation $f=T_{1}+\sum_{\nu=0}^{\infty}\left(T\left(2^{\nu+1}\right)-T\left(2^{\nu}\right)\right)$, convergent in $L^{p}$. However, by Remark 2.1 and the triangle inequality

$$
\left\|T\left(2^{\nu+1}\right)-T\left(2^{\nu}\right)\right\|_{\infty} \leq 6 \cdot 2^{\nu / p}\left\|T\left(2^{\nu+1}\right)-T\left(2^{\nu}\right)\right\|_{p} \leq 24 \cdot 2^{\nu / p} E\left(2^{\nu} ; p ; f\right) ;
$$

so that, if we assume $\int_{0}^{1} \omega(h ; p ; f) h^{-1-1 / p} d h<\infty$, then the series representing $f$ converges in $L^{\infty}$. In particular, after contingent modification on a set of measure zero, $f$ is $2 \pi$-periodic and continuous on $\mathbb{R}$.

Lebesgue's test $\left[22\right.$, p. 65] is expressed in terms of $\varphi_{x}(t) \doteq f(x+t)-$ $2 f(x)+f(x-t)$. It asserts that if $f \in L^{1}[0,2 \pi]$ and, as $h \rightarrow 0^{+}$,

(i) $h^{-1} \int_{0}^{h}\left|\varphi_{x}(t)\right| d t \rightarrow 0$ for each $x \in[0,2 \pi]$, and

(ii) $\int_{h}^{1}\left|\varphi_{x}(t+h)-\varphi_{x}(t)\right| t^{-1} d t \rightarrow 0$ uniformly with respect to $x \in[0,2 \pi]$, then the Fourier series of $f$ is uniformly convergent on $[0,2 \pi]$. Evidently continuity of $f$ ensures that (i) holds. As to the validity of (ii) for a function $f$ satisfying Garsia's criterion (compare [22, Sections 10, 11 of Chapter II]), observe that $\omega\left(h ; p ; \varphi_{x}\right) \leq 2 \omega(h ; p ; f)$. (Indeed, by Minkowski's 
inequality

$$
\begin{aligned}
& \Omega\left(h ; p ; \varphi_{x}\right) \leq\left\{(1 / 2 \pi) \int_{0}^{2 \pi}|f(x+t+h)-f(x+t)|^{p} d t\right\}^{1 / p} \\
& \left.\quad+\left\{(1 / 2 \pi) \int_{0}^{2 \pi}|f(x-t-h)-f(x-t)|^{p} d t\right\}^{1 / p}=2 \Omega(h ; p ; f)\right) .
\end{aligned}
$$

Therefore, for $1<p<\infty$, by Hölder's inequality,

$$
\begin{aligned}
\int_{h}^{1}\left|\varphi_{x}(t+h)-\varphi_{x}(t)\right| t^{-1} d t \\
\quad \leq\left\{\int_{0}^{1}\left|\varphi_{x}(t+h)-\varphi_{x}(t)\right|^{p} d t\right\}^{1 / p}\left\{\int_{h}^{1} t^{p /(p-1)} d t\right\}^{1-1 / p} \\
\quad \leq A_{p} \omega\left(h ; p ; \varphi_{x}\right) h^{-1 / p} \\
\quad \leq A_{p} \omega(h ; p ; f) h^{-1 / p} \rightarrow 0 \text { as } h \rightarrow 0^{+} .
\end{aligned}
$$

This provides a proof, of the kind sought by Garsia, that a $2 \pi$-periodic $f$ has a uniformly convergent Fourier series if $\int_{0}^{1} \omega(h ; p ; f) h^{-1-1 / p} d h<\infty$.

REMARK 2.2. In [8] and in [7] Garsia's criterion was formulated in terms of the modulus $\Omega$, whereas we have employed the more tractable modulus $\omega$. We have not, however, relinquished any functions by adopting $\omega$. To see this we write $\rho \doteq-1-1 / p$ and $\varphi(h) \doteq \int_{0}^{h} \Omega(\lambda ; p ; f) d \lambda$. Garsia proved $[8, \mathrm{p} .91]$ that $\omega(h ; p ; f) \leq 10 h^{-1} \varphi(h)$. We are assuming that $\int_{0}^{1} h^{\rho} d \varphi(h)$ is finite. By partial integration, $\int_{0}^{1} h^{\rho} \omega(h ; p ; f) d h \leq 10 \int_{0}^{1} h^{\rho-1} \varphi(h) d h=$ $10 \rho^{-1}\left[h^{\rho} \varphi(h)\right]_{0}^{1}-10 \rho^{-1} \int_{0}^{1} h^{\rho} d \varphi(h)$. Because $\rho<0, h^{\rho} \varphi(h) \leq \int_{0}^{h} \lambda^{\rho} d \varphi(\lambda)$; thus the integral $\int_{0}^{1} h^{\rho} \omega(h ; p ; f) d h$ must also be finite.

\section{Preliminaries}

For positive functions (or functionals) $\Gamma$ and $\Delta, \Gamma \approx \Delta$ means $\Gamma=O(\Delta)$ and $\Delta=O(\Gamma)$, while $\Gamma \sim \Delta$ means $\Gamma / \Delta \rightarrow 1$. Generic constants are denoted by $A$.

A continuous function $L(t)>0$ is slowly varying if $L(c t) \sim L(t)$ as $t \rightarrow \infty$, for each $c>0$. This relation then holds uniformly for $c$ in any compact subset of $(0, \infty)$. A slowly varying function $L$ satisfies $L(t)=o\left(t^{\alpha}\right)$ if $\alpha>0$. Zygmund defines, more restrictedly, a slowly varying function $L$ [22, p. 186] as one for which $t^{-\alpha} L(t) \downarrow 0$ and $t^{\alpha} L(t) \uparrow \infty$ for each $\alpha>0$. Every slowly varying function is asymptotic to one of Zygmund's type. If $L$ is slowly varying and $\delta>0, \sum_{1}^{n} L(k) k^{\delta-1} \sim \delta^{-1} L(n) n^{\delta}$ and $\sum_{n}^{\infty} L(k) k^{-\delta-1} \sim \delta^{-1} L(n) n^{-\delta}$ (similarly for integrals). Prime examples of 
slowly varying functions are sums of products of iterated logarithms. See [22] and [16] for references.

The periodic Besov space $\Lambda(\alpha, p, q)$ consists of those $2 \pi$-periodic $f \in$ $L^{p}[0,2 \pi]$ (or $f \in C[0,2 \pi]$ when $p=\infty$ ) for which

$$
\int_{0}^{\pi} h^{-1-\alpha q} \omega^{q}(h ; p ; f) d h<\infty .
$$

Herein $0<\alpha \leq 1,1 \leq p \leq \infty, 0<q<\infty$. For $q=\infty$, the inequality is replaced by $\omega(h ; p ; f)=O\left(h^{\alpha}\right)$. Thus $\Lambda(\alpha, p, \infty)=\operatorname{Lip}(\alpha, p)$, in the notation of Hardy-Littlewood. The spaces with $q<1$ are not normable, but here they need no special consideration. Were $\pi$ replaced by another upper limit in the defining integral, $\Lambda(\alpha, p, q)$ would be unaltered. Occasionally, 1 or $\infty$ is more convenient.

Evidently $\Lambda(\alpha, p, q)$ shrinks if $\alpha$ is increased or $p$ is increased. As in Section 2 we see that every $f \in \Lambda(\alpha, p, q)$ with $q<\infty$ satisfies $\omega(h ; p ; f)$ $=o\left(h^{\alpha}\right)$. It follows that $\Lambda(\alpha, p, q)$ expands when $q$ is increased and that $\Lambda\left(\alpha_{2}, p, q_{2}\right) \subset \Lambda\left(\alpha_{1}, p, q_{1}\right)$ for all $q_{1}, q_{2}$ provided $\alpha_{1}<\alpha_{2}$. Thus the third index serves to specify significant spaces between $\operatorname{Lip}\left(\alpha_{2}, p\right)$ and $\operatorname{Lip}\left(\alpha_{1}, p\right)$. For example, while $\Lambda\left(p^{-1}, p, q\right)$ contains discontinuous functions if $q>1$ (see Section 4), all functions in $\Lambda\left(p^{-1}, p, 1\right)$ possess uniformly convergent Fourier series. We are concerned mainly with these spaces. They enlarge with increasing $p$, a dependence upon $p$ more subtle than the inclusions cited above. It is a special case of a general theorem. See [7, p. 363] or Theorem 6.1 infra.

The remainder of this section sets out some needed facts for convenient reference.

3.1. We let $\Lambda^{*}(\alpha, p, q)$ denote the space of functions $f$ for which $\omega^{*}(h ; p ; f)$ appears in the defining inequality. $\left(\omega^{*}\right.$ is the modulus of continuity defined in terms of a second difference). Here it is appropriate to allow $0<\alpha<2$ (see [5, p. 67]). Evidently, $\omega^{*} \leq 2 \omega$; but so long as $\alpha<1, \Lambda^{*}(\alpha, p, q)=\Lambda(\alpha, p, q)$ (see [4, p. 229]). However, for $q<\infty, \Lambda(1, p, q)$ consists of constant functions only [22, p. 45], whereas $\Lambda^{*}(1, p, q) \supset C^{(2)}$ (see 3.2). It is also shown in [4, p. 228] that the same space $\Lambda(\alpha, p, q)$ results when $\Omega$ is employed in the defining integral instead of $\omega$ (compare Remark 2.2 concerning $\Lambda\left(p^{-1}, p, 1\right)$ ) .

3.2. The first Jackson approximation theorem [5, p. 97] asserts that if $1 \leq p \leq \infty$, then $E(n ; p ; f) \leq 18 \omega^{*}\left(n^{-1} ; p ; f\right)$. By virtue of this inequality, for $0<\alpha<2,1 \leq p \leq \infty, 0<q \leq \infty$, the functions belonging to $\Lambda^{*}(\alpha, p, q)$ satisfy (compare the proof of Theorem 5.1) $\left\{n^{\alpha-1 / q} E(n ; p ; f)\right\}$ $\in l_{q}$. Conversely, these inclusions imply that $f \in \Lambda^{*}(\alpha, p, q)$. (See [3, 
Section 1] or [2, Theorem 7.2.4 and exercise 7, p. 188]; we provide a simpler proof consonant with the methods of this paper in Lemma 3.12). This fact verifies, as we have mentioned, that $C^{(2)} \subset \Lambda^{*}(\alpha, p, q)$ for $\alpha<2$, because $E(n ; \infty ; f)=O\left(n^{-r} \omega^{*}\left(n^{-1} ; \infty ; f^{(r)}\right)\right)$ if $f \in C^{(r)}[5$, p. 97]

3.3. For $f \in C, \omega(h ; \infty ; f) \leq A h \sum_{1}^{1 / h} E(\nu ; \infty ; f)$ and $\omega^{*}(h ; \infty ; f) \leq$ $A h^{2} \sum_{1}^{1 / h} \nu E(\nu ; \infty ; f)$. Compare with 3.2. The proof can be found in [14, p. 59].

3.4. For certain lacunary series $E(n ; \infty)$ is precisely expressible in terms of the coefficients. Suppose $\left\{a_{k}\right\}$ is a non-negative sequence in $l_{1}$. Let $g(t) \doteq \sum_{0}^{\infty} a_{k} \cos 3^{k} t$. Then [15, p. 73] $E(m ; \infty ; g)=\sum_{n+1}^{\infty} a_{k}$, when $3^{n} \leq m<3^{n+1}$.

3.5. Suppose $1<p<\infty, g \in L^{1}$, and $g$ is even or odd with $\hat{g}(k) \downarrow 0$ as $k \rightarrow \infty$. Then $g \in L^{p}$ and $\omega\left(k^{-1} ; p ; f\right)=O\left(k^{1-1 / p} \hat{g}(k)\right)$, provided that $\sum_{1}^{n} k^{1-1 / p} \hat{g}(k)=O\left(n^{2-1 / p} \hat{g}(n)\right)$ and $\sum_{n+1}^{\infty} k^{p-2}(\hat{g}(k))^{p}=O\left(n^{p-1}(\hat{g}(n))^{p}\right)$. See [1]. The properties of a slowly varying function $L(t)$ stated above verify that these conditions are satisfied if $\hat{g}(k) \doteq k^{-a} L(k)$ and $1<a+1 / p<2$.

3.6. A simple weakened converse of the estimate in 3.5 is valid for all $f \in L^{1}$ and $1 \leq p \leq \infty$ [22, pp. 45 and 47]: $|\hat{f}(n)| \leq(1 / 2) \omega(\pi /|n| ; p ; f)$ and $|\hat{f}(n)| \leq(1 / 4) \omega^{*}(\pi /|n| ; p ; f)$.

3.7. If $c>0, \omega(c h ; p ; f) \leq(c+1) \omega(h ; p ; f)$ and $\omega^{*}(c h ; p ; f) \leq$ $(c+1)^{2} \omega^{*}(h ; p ; f)$. If $c \in \mathbb{Z}^{+}, c+1$ can be replaced by $c[5$, p. 67].

3.8. Suppose $\hat{f}(0)=0$. The theorem of $\mathbf{M}$. Riesz guarantees that for each $p \in(1, \infty)$ there exists $c_{p}$ such that for real $f \in L^{p},\|\tilde{f}\|_{p} \leq c_{p}\|f\|_{p}$; here $\tilde{f}$ denotes the conjugate function. It follows [12, pp. 48, 49] that for complex $f \in L^{p},\|s(n ; f)\|_{p} \leq\left(1+c_{p}\right)\|f\|_{p}$, where $s(n ; f)$ denotes the $n$th Fourier sum. Thus, if $T$ is a trigonometric polynomial of degree $\leq n$,

$$
\|s(n ; f)-f\|_{p} \leq\left(2+c_{p}\right)\|T-f\|_{p} .
$$

Hence,

$$
\|s(n ; f)-f\|_{p} \leq\left(2+c_{p}\right) E(n ; p ; f) .
$$

In particular, $s(n ; f) \rightarrow f$ in $L^{p}$. For each $p \in(1, \infty)$ there exists a smallest value of $c_{p}$. Henceforth we let $c_{p}$ denote that smallest value. It is known [18] that when $p \leq 2, c_{p}=\tan (\pi / 2 p)$; while if $p \geq 2, c_{p}=$ $\cot (\pi / 2 p)$.

3.9. Although $s(n ; f)$ need not converge in $L^{1}$ for $f \in L^{1}$, nor in $L^{\infty}$ for $f \in C$, the de la Vallée-Poussin sums $\tau(n ; f)$ do. $\tau$ is defined, in terms 
of the Cesaro $(C, 1)$ means $\sigma$, by $\tau(n ; f) \doteq 2 \sigma(2 n-1 ; f)-\sigma(n-1 ; f)$, where $\sigma(n ; f) \doteq(n+1)^{-1} \sum_{0}^{n} s(k ; f)$. Then $([5$, p. 108] and [22, p. 115])

$$
\|\tau(n ; f)-f\|_{p} \leq 4 E(n ; p ; f)
$$

for $f \in L^{p}(1 \leq p<\infty)$ and for $f \in C \quad(p=\infty)$. The degree of $\tau(n ; f)$ is $2 n-1$. If the degree of a polynomial $T$ does not exceed $n$, then $\tau(n ; T)=$ $T$.

3.10. An integral version of the Olivier-Hardy theorem takes the following form. Suppose that $\varphi(\lambda)$ and $\psi(\lambda)$ are positive and $\psi$ is increasing on $(0,1)$. Suppose also that $\Phi(\lambda) \doteq \int_{\lambda}^{1} \varphi$ exists for $0<\lambda \leq 1$, that $\Phi(\lambda) \rightarrow \infty$ as $\lambda \rightarrow 0^{+}$, and that $\int_{0}^{1} \varphi \psi<\infty$. Then $\psi(\lambda) \Phi(\lambda) \rightarrow 0$ as $\lambda \rightarrow 0^{+}$. (For plainly $\psi(\lambda) \rightarrow 0$ as $\lambda \rightarrow 0^{+}$. Further, there exists $\delta>0$ such that $0<$ $\lambda \leq \delta$ implies that $\psi(\lambda)[\Phi(\lambda)-\Phi(\delta)] \leq \int_{\lambda}^{\delta} \varphi \psi<\varepsilon$. Hence $\psi(\lambda) \Phi(\lambda)<$ $\varepsilon+\psi(\lambda) \Phi(\delta)<2 \varepsilon$ for all small $\lambda>0$.) The series analog of this shows that if $\left\{a_{n}\right\}$ is quasi-monotonic and belongs to $l_{1}$, then $a_{n}=o\left(n^{-1}\right)$. (Quasimonotonic means that $n^{-\alpha} a_{n} \downarrow 0$ for some $\alpha>0$.)

Lemma 3.11. Suppose $a_{n} \geq 0$ and $q \geq 1$. Then [10, p. 255] if $c>1$,

while if $c<1$ and $\left\{a_{n}\right\} \in l_{1}$,

$$
\sum_{1}^{\infty} n^{-c}\left(\sum_{1}^{n} a_{k}\right)^{q} \leq A \sum_{1}^{\infty} n^{q-c} a_{n}^{q}
$$

$$
\sum_{1}^{\infty} n^{-c}\left(\sum_{n}^{\infty} a_{k}\right)^{q} \leq A \sum_{1}^{\infty} n^{q-c} a_{n}^{q} .
$$

Furthermore, both inequalities hold when $0<q<1$ provided $\left\{a_{n}\right\}$ is quasimonotonic [16, p. 583].

Lemma 3.12. Suppose $0<\alpha<2,1 \leq p \leq \infty, 0<q<\infty$ and $f \in L^{p}$. Then

$$
\sum_{1}^{\infty} n^{\alpha q-1}\left(\omega^{*}\left(n^{-1} ; p ; f\right)\right)^{q} \leq A_{\alpha, p, q} \sum_{1}^{\infty} n^{\alpha q-1} E^{q}(n ; p ; f),
$$

and

$$
\sup n^{\alpha} \omega^{*}\left(n^{-1} ; p ; f\right) \leq A_{\alpha, p} \sup n^{\alpha} E(n ; p ; f) .
$$

Consequently, there exist $A_{\alpha, p, q}$ and $A_{\alpha, p, q}^{\prime}$ such that

$$
\begin{aligned}
A_{\alpha, p, q}\left\|\left\{n^{\alpha-1 / q} E(n ; p ; f)\right\}\right\|_{l_{q}} & \leq\left\{\int_{0}^{1} h^{-1-\alpha q}\left(\omega^{*}(h ; p ; f)\right)^{q} d h\right\}^{1 / q} \\
& \leq A_{\alpha, p, q}^{\prime}\left\|\left\{n^{\alpha-1 / q} E(n ; p ; f)\right\}\right\|_{l_{q}}
\end{aligned}
$$

If $q=\infty$, replace the middle term by $\sup \left\{h^{-\alpha} \omega^{*}(h ; p ; f): 0<h \leq 1\right\}$. 
Proof. We utilize 3.3 (valid, in fact, with $\infty$ replaced by any $p \in[1, \infty]$; compare [19]) and Lemma 3.11 to obtain, for $0<q<\infty$,

$$
\begin{aligned}
\sum_{1}^{\infty} n^{\alpha q-1}\left(\omega^{*}\left(n^{-1} ; p ; f\right)\right)^{q} & \leq A \sum_{1}^{\infty} n^{\alpha q-1}\left(n^{-2} \sum_{1}^{n} \nu E(\nu ; p ; f)\right)^{q} \\
& \leq A \sum_{1}^{\infty} n^{q+(\alpha q-1-2 q)} n^{q} E^{q}(n ; p ; f)
\end{aligned}
$$

provided $c \doteq-\alpha q+1+2 q>1$; that is, provided $\alpha<2$. This accounts for the first inequality. The second is simpler. Let $M_{f} \doteq \sup n^{\alpha} E(n ; p ; f)$ (which we may assume to be finite); so that $E(\nu ; p ; f) \leq M_{f} \nu^{-\alpha}$. Accordingly, by 3.3 ,

$$
\begin{aligned}
n^{\alpha} \omega^{*}\left(n^{-1} ; p ; f\right) & \leq A_{p} n^{\alpha-2} \sum_{1}^{n} \nu E(\nu ; p ; f) \\
& \leq A_{p} n^{\alpha-2} M_{f} \sum_{1}^{n} \nu^{1-\alpha} \leq A_{\alpha, p} M_{f}
\end{aligned}
$$

as asserted.

When $q<\infty$ inequalities similar to those at the end of the proof of Theorem 5.1 substantiate the left norm inequality. Further, the only relevant inequality in that proof which is not obviously reversible is proved to be so by the first part of the lemma. When $q=\infty$ the analogous sup inequalities are evident in virtue of the sup inequality in the first part of the lemma.

\section{Comparison of criteria for uniform convergence}

As well as serving to prove Garsia's Theorem Lebesgue's test provides an economical demonstration [11, Theorem 59] of the Dini-Lipschitz theorem, which asserts that a $2 \pi$-periodic function possesses a uniformly convergent Fourier series when $\omega(h ; \infty ; f)=o\left(|\log h|^{-1}\right)$. Garsia's Theorem asserts that if $f \in \Lambda\left(p^{-1}, p, 1\right)$, where $1 \leq p<\infty$, then its Fourier series is uniformly convergent. Hobson's criterion (see Section 1) can be interpreted formally as asserting that $f \in \Lambda(0, \infty, 1) .3 .10$ shows that such an $f$ must satisfy the Dini-Lipschitz condition. The converse is not true since the function $\varphi(t) \doteq|(\log t)(\log |\log t|)|^{-1}$ is increasing and concave on some interval $(0, \delta]$; hence $[14$, p. 44] $g(t) \doteq \varphi(|\delta t / \pi|)$ on $[-\pi, \pi]$ has the modulus of continuity $\omega(h ; \infty ; g)=g(h) \quad(0<h \leq \pi)$.

We indicated in Section 3 that Garsia's spaces $\Lambda\left(p^{-1}, p, 1\right)$ enlarge 
with increasing $p \in[1, \infty)$. It is instructive to observe the inclusion of the Weierstrass-Hardy functions $W_{a}(t) \doteq \sum_{0}^{\infty} 3^{-a k} \cos 3^{k} t \quad(0<a \leq 1)$ in $\Lambda\left(p^{-1}, p, 1\right)$. By 3.6, for $1 \leq p \leq \infty, \omega^{*}\left(3^{-k} ; p ; W_{a}\right) \geq A 3^{-a k}$, hence by $3.7, \omega^{*}\left(h ; p, W_{a}\right) \geq A h^{a}$. The reverse inequality is valid for $0<a \leq 1$ [22, p. 47]. Accordingly, when $0<a<1, W_{a} \in \Lambda\left(p^{-1}, p, 1\right)$ if and only if $p>1 / a$, while $W_{1} \in \Lambda^{*}\left(p^{-1}, p, 1\right)$ if and only if $p>1$. We are therefore in possession of functions which, for $\infty>r>p \geq 1$, belong to $\Lambda\left(r^{-1}, r, 1\right)$ but do not belong to $\Lambda\left(p^{-1}, p, 1\right)$; and we see further that the inclusion (compare Remark 6.2) $\Lambda^{*}(1,1,1) \subset \Lambda\left(p^{-1}, p, 1\right)$, for $1<p<\infty$, also is proper. Functions in $\Lambda\left(r^{-1}, r, 1\right)$ which do not belong to $\Lambda\left(p^{-1}, p, 1\right)$ also are constructed in $[7$, p. 365] when $2 \leq p<r$.

Since $\Lambda\left(p^{-1}, p, 1\right)$ expands with increasing $p$ it would not be surprising if Garsia's Theorem were subsumed by Hobson's Theorem. To establish that this is not so we exhibit a function that belongs to $\Lambda\left(p^{-1}, p, 1\right)$ for all $p \in(1, \infty)$ but fails even to satisfy the Dini-Lipschitz condition. If $1<p<\infty$, the even function $g$ with coefficients $\hat{g}(n) \doteq|n|^{-1} L(|n|)$ satisfies $\omega\left(n^{-1} ; p ; g\right)=O\left(n^{1-1 / p} \hat{g}(n)\right)$ as in 3.5 ; so $\omega(h ; p ; g) h^{-1-1 / p}$ $=O\left(h^{-1} L\left(h^{-1}\right)\right)$. Accordingly,

$$
g \in \Lambda\left(p^{-1}, p, 1\right) \text { if } \int_{1}^{\infty} x^{-1} L(x) d x \equiv \int_{0}^{1} h^{-1} L\left(h^{-1}\right) d h<\infty ;
$$

and we assume $L$ to be so specified as well as slowly varying. By [22, p. 188 , Theorem (2.15)], $\omega(h ; \infty ; g) \geq A \int_{1 / h}^{\infty} x^{-1} L(x) d x$. So plainly, $L$ can be chosen so that $\omega(h ; \infty ; g)$ approaches zero less rapidly than, for instance, the reciprocal of any specified iterate of log; hence $g$ will be far from meeting the Dini-Lipschitz criterion. (A propos, if the small $o$ is modified to $O$ in the Dini-Lipschitz condition there exists a function that satisfies the relaxed hypothesis but possesses a Fourier series that diverges on a set dense in $[0,2 \pi]$; see $[22$, p. 303$]$.)

In the other direction, there are functions that satisfy Hobson's criterion, yet do not belong to $\Lambda\left(p^{-1}, p, 1\right)$ for any $p<\infty$. For example, 3.6, applied to the function $W_{0}(t) \doteq \sum_{0}^{\infty} L\left(3^{k}\right) \cos 3^{k} t$, with $L$ a slowly varying function, shows that

$$
\omega\left(3^{-k} ; p ; W_{0}\right) \geq A \hat{W}_{0}\left( \pm 3^{k}\right) \geq A L\left(3^{k}\right) \quad \text { for } 1 \leq p \leq \infty .
$$

Hence, since $L$ is slowly varying, by $3.7, \omega\left(h ; p ; W_{0}\right) \geq A L\left(h^{-1}\right)$. It follows that $\int_{0}^{1} h^{-1-1 / p} \omega\left(h ; p ; W_{0}\right) d h$ diverges for every slowly varying function $L$; that is, $W_{0}$ belongs to no space $\Lambda\left(p^{-1} ; p, 1\right)$ for $p<\infty$. On 
the other hand, by Lemma 4.1 infra, $W_{0}$ satisfies Hobson's criterion if $\int_{0}^{1} h^{-1} R(h) d h<\infty$. By Fubini's theorem this inequality is equivalent to $L$ satisfying $\int_{1}^{\infty} u^{-1} L(u) \int_{1 / u}^{1} h^{-1} d h=\int_{1}^{\infty} u^{-1} L(u) \log u d u<\infty$; and there is no dearth of such slowly varying functions to provide the desired example.

LEMMA 4.1. Let $W_{0}(t) \doteq \sum_{0}^{\infty} L\left(3^{k}\right) \cos 3^{k} t$. Then $\omega\left(h ; \infty ; W_{0}\right) \approx R(h)$ $\doteq \int_{1 / h}^{\infty} x^{-1} L(x) d x$.

Proof. Let $T(n) \doteq \sum_{n}^{\infty} k^{-1} L(k) \sim R\left(n^{-1}\right)$ (See [22, p. 188]). Observe that (a) condensation is valid for sums of slowly varying terms, (b) $\sum_{1}^{n} L(k)=O(n L(n))$, and (c) $L(n)=o(T(n))$ (see [22, p. 188] or [16]). We now employ $3.3,3.4$, interchange of the order of summation, and (a), (b), and (c) to conclude that

$$
\begin{aligned}
& \omega\left(3^{-n} ; \infty ; W_{0}\right) \leq A 3^{-n} \sum_{k=1}^{3^{n}} E\left(k ; \infty ; W_{0}\right) \\
& \quad=2 A \cdot 3^{-n} \sum_{\nu=0}^{n} 3^{\nu} E\left(3^{\nu} ; \infty ; W_{0}\right) \leq A 3^{-n} \sum_{\nu=0}^{n} 3^{\nu} \sum_{k=\nu}^{\infty} L\left(3^{k}\right) \\
& =A \cdot 3^{-n} \sum_{k=0}^{n} L\left(3^{k}\right) \sum_{\nu=0}^{k} 3^{\nu}+A \cdot 3^{-n} \sum_{k=n+1}^{\infty} L\left(3^{k}\right) \sum_{\nu=0}^{n} 3^{\nu} \\
& \quad \leq A 3^{-n} \sum_{k=0}^{n} 3^{k} L\left(3^{k}\right)+A \sum_{k=n}^{\infty} L\left(3^{k}\right) \leq A 3^{-n} \sum_{\nu=1}^{3^{n}} L(\nu)+A \sum_{\nu=3^{n}}^{\infty} \nu^{-1} L(\nu) \\
& \quad \leq A\left(L\left(3^{n}\right)+T\left(3^{n}\right)\right) \\
& =A\left[o\left(T\left(3^{n}\right)\right)+T\left(3^{n}\right)\right] \leq A T\left(3^{n}\right) \leq A R\left(3^{-n}\right) .
\end{aligned}
$$

Thus by 3.7 and the monotonicity of $\omega$ and $R, \omega\left(h ; \infty ; W_{0}\right) \leq A R(h)$. The reverse inequality requires only 3.2 and 3.4 :

$$
\begin{aligned}
\omega\left(3^{-n} ; \infty ; W_{0}\right) & \geq A E\left(3^{n} ; \infty ; W_{0}\right) \\
& =A \sum_{n+1}^{\infty} L\left(3^{k}\right) \geq A \sum_{\nu=3^{n+1}}^{\infty} \nu^{-1} L(\nu) \approx R\left(3^{-n-1}\right),
\end{aligned}
$$

from which the required inequality readily follows.

Since the portion of our proof of Garsia's Theorem that involves condition (ii) of Lebesgue's test applies equally to functions in $\Lambda\left(p^{-1}, p, q\right)$ with $q<$ $\infty$, it is of interest that, when $q>1, \Lambda\left(p^{-1}, p, q\right)$ contains discontinuous 
functions. For example [22, p. 253], define $g$ by $\hat{g}(n)=(|n| \log |n|)^{-1}$ for $|n|>1$. By 3.5, $g \in L^{p}$ and $\omega\left(n^{-1} ; p ; g\right)=O\left(n^{1-1 / p} n^{-1}(\log n)^{-1}\right)$; so $\omega(h ; p ; g)=O\left(h^{1 / p}|\log h|^{-1}\right)$. Accordingly,

$$
\int_{0}^{1} \omega^{q}(h ; p ; g) h^{-1-q / p} d h \leq A \int_{0}^{1} h^{-1}|\log h|^{-q} d h<\infty \text { if } q>1 ;
$$

that is, $g \in \Lambda\left(p^{-1}, p, q\right)$. However, if $g$ were (equivalent to a function that is) continuous at $0, \sum \hat{g}(n)$ would be $(C, 1)$ summable [22, p. 89], hence convergent, since $\hat{g}(n) \geq 0$.

REMARK 4.2. The substance of the last sentence verifies that the Fourier series of an even function $g$ that has positive coefficients is uniformly convergent if and only if it is absolutely convergent; and, if $g$ satisfies the conclusion of 3.5, this occurs if and only if $g \in \Lambda\left(p^{-1}, p, 1\right)$ for some/every $p \in(1, \infty)$ (the latter because $A \sum \hat{g}(n) \geq \sum n^{1 / p-1} \omega\left(n^{-1} ; p ; g\right) \approx$ $\left.\int_{0}^{1} h^{-1 / p-1} \omega(h ; p ; g) d h\right)$.

\section{A quantitative version of Garsia's Theorem}

By appeal to the M. Riesz theorem (3.8) we can modify the proof in Section 2 to estimate the rate of convergence of the Fourier partial sums $s(n ; f)=$ $s(n)$.

TheOREM 5.1. Suppose $1<p<\infty$ and $f \in \Lambda\left(p^{-1}, p, 1\right)$. Then, for $n \geq 1$,

$$
\|s(n ; f)-f\|_{\infty} \leq\left(c_{p}+2\right) 3456 \int_{0}^{1 / n} h^{-1-1 / p} \omega^{*}(h ; p ; f) d h
$$

Proof. We may assume that $\hat{f}(0)=0$ since neither $s(n ; f)-f$ nor $\omega^{*}$ is altered by addition of a constant to $f$. For a specified $n>0$, let $k$ satisfy $2^{k-1} \leq n<2^{k}$, and write, as in Section $2, f-s(n)=s\left(2^{k}\right)-$ $s(n)+\sum_{\nu=k}^{\infty}\left[s\left(2^{\nu+1}\right)-s\left(2^{\nu}\right)\right]$, convergent in $L^{p}$. For the nonce, let $K=$ $6 \cdot 2^{1 / p}\left(2+c_{p}\right)$. Again, we use the estimate $\|T\|_{\infty} \leq 3 n^{1 / p}\|T\|_{p}, 3.8$ and the triangle inequality, 3.2 , the fact that by 3.7 ,

$$
\begin{aligned}
\sum_{j=2^{\nu+1}}^{2^{\nu+2}-1} j^{1 / p-1} \omega^{*}\left(j^{-1} ; p\right) & \geq 2^{\nu+1} 2^{(\nu+2)(1 / p-1)} \omega^{*}\left(2^{-\nu-2} ; p\right) \\
& \geq 2^{-1+2 / p+\nu / p} \cdot 4^{-2} \omega^{*}\left(2^{-\nu} ; p\right),
\end{aligned}
$$


and the fact that $2^{k}>n$ to conclude that

$$
\begin{aligned}
\|f-s(n)\|_{\infty} \leq & 3 \cdot 2^{k / p} \cdot 2\left(2+c_{p}\right) E\left(2^{k-1} ; p\right) \\
& +3 \cdot 2^{1+1 / p}\left(2+c_{p}\right) \sum_{\nu=k}^{\infty} 2^{\nu / p} E\left(2^{\nu} ; p\right) \\
= & K \sum_{\nu=k-1}^{\infty} 2^{\nu / p} E\left(2^{\nu} ; p\right) \leq 18 K \sum_{\nu=k-1}^{\infty} 2^{\nu / p} \omega^{*}\left(2^{-\nu} ; p\right) \\
\leq & 18 K \cdot 32 \cdot 2^{-1 / p} \sum_{\nu=k-1}^{\infty} \sum_{j=2^{\nu+1}}^{2^{\nu+2}-1} j^{1 / p-1} \omega^{*}\left(j^{-1} ; p\right) \\
= & 2^{7} \cdot 3^{3}\left(2+c_{p}\right) \sum_{j=2^{k}}^{\infty} j^{1 / p-1} \omega^{*}\left(j^{-1} ; p\right) \\
< & 2^{7} \cdot 3^{3}\left(2+c_{p}\right) \int_{n}^{\infty} t^{1 / p-1} \omega^{*}\left(t^{-1} ; p\right) d t \\
= & 2^{7} \cdot 3^{3}\left(2+c_{p}\right) \int_{0}^{1 / n} h^{-1-1 / p} \omega^{*}(h ; p) d h .
\end{aligned}
$$

REMARK 5.4. (i) The seemingly facetious 3456 that appears in (5.2) has been retained only to emphasize the determinability of a specific bound. (ii) I do not know whether $(5.2)$ is valid for all functions in $\Lambda^{*}(1,1,1)$. (The proof fails when $p=1$ with the failure of Riesz's theorem.) Notwithstanding, every $f \in \Lambda^{*}(1,1,1)$ has a uniformly convergent Fourier series as a consequence of Remark 6.2 infra. This also follows from the Dirichlet-Jordan theorem [22, p. 59].

REMARK 5.5. Theorem 5.1 cannot be improved to assert that

$$
\|f-s(n ; f)\|_{\infty}=o\left(\int_{0}^{1 / n} h^{-1-1 / p} \omega(h ; p ; f) d h\right) .
$$

For, by 3.5 , when $1<p<\infty$ and $1<\gamma+1 / p<2$ the function $g_{\gamma}(t) \doteq$ $\sum_{1}^{\infty} n^{-\gamma} \cos n t$ satisfies $\omega\left(n^{-1} ; p ; g_{\gamma}\right)=O\left(n^{1-1 / p} \hat{g}_{\gamma}(n)\right)$; hence

$$
\omega\left(h ; p ; g_{\gamma}\right)=O\left(h^{\gamma-1+1 / p}\right) \text {. }
$$

The validity of the $o$ estimate would therefore entail

$$
\left\|g_{\gamma}-s\left(n ; g_{\gamma}\right)\right\|_{\infty}=o\left(\int_{0}^{1 / n} h^{\alpha-2} d h\right)=o\left(n^{1-\gamma}\right),
$$


from which $E\left(n ; \infty ; g_{\gamma}\right)=o\left(n^{1-\gamma}\right)$. In turn this implies, via 3.3, that

$$
\omega\left(h ; \infty ; g_{\gamma}\right)=O\left(h \sum_{n=1}^{1 / h} o\left(n^{1-\gamma}\right)\right)=o\left(h^{\gamma-1}\right),
$$

since $\gamma<2$. A contradiction is now at hand; for by [22, p. 229, Example 11], if $1<\gamma<2$ then, as $t \rightarrow 0^{+}, g_{\gamma}(t)-g_{\gamma}(0) \sim A_{\gamma} t^{\gamma-1}$, where $A_{\gamma} \neq 0$; so in fact, $\omega\left(h ; \infty ; g_{\gamma}\right) \neq o\left(h^{\gamma-1}\right)$. Thus we need only stipulate that $\gamma$ satisfy $1<\gamma<2-1 / p$.

Corollary 5.6. If $f \in \Lambda\left(p^{-1}, p, 1\right)$ for some $p \in(1, \infty)$, then

$$
\begin{aligned}
\|f-\hat{f}(0)\|_{\infty} \leq & 3456\left(2+c_{p}\right) \int_{0}^{1} h^{-1-1 / p} \omega^{*}(h ; p ; f) d h \\
& +8 \pi^{2} \int_{1}^{\pi} h^{-1-1 / p} \omega^{*}(h ; p ; f) d h \\
\leq & A_{p} \int_{0}^{\pi} h^{-1-1 / p} \omega^{*}(h ; p ; f) d h .
\end{aligned}
$$

Proof. The value $n=1$ in (5.2) yields, by the triangle inequality, $\|f-\hat{f}(0)\|_{\infty} \leq|\hat{f}(1)|+|\hat{f}(-1)|+3456\left(2+c_{p}\right) \int_{0}^{1} h^{-1-1 / p} \omega^{*}(h ; p ; f) d h$.

From 3.6,

while by 3.7 ,

$$
|\hat{f}(1)|+|\hat{f}(1)| \leq(1 / 2) \omega^{*}(\pi ; p ; f) ;
$$

$$
\int_{1}^{\pi} h^{-1-1 / p} \omega^{*}(h ; p ; f) d h \geq \omega^{*}(\pi ; p ; f) / 16 \pi^{2} .
$$

REMARK 5.7. Corollary 5.6 contains a key result in [8], from which Garsia deduces several interesting theorems, including the inequality referred to in the first sentence of Section 1.

COROLlary 5.8. Suppose $f$ is absolutely continuous and $f^{\prime} \in L^{p}$, where $1<p<\infty$. Then $\|f-s(n ; f)\|_{\infty} \leq A_{p} \int_{0}^{1 / n} h^{-1 / p} \omega\left(h ; p ; f^{\prime}\right) d h$.

Proof. If $f$ is absolutely continuous and $f^{\prime} \in L^{p} \quad(1 \leq p \leq \infty)$ then $\omega^{*}(h ; p ; f) \leq h \omega\left(h ; p ; f^{\prime}\right)$. (For $p=\infty$ see $[14$, p. 47$]$; the proof for other values of $p$ is similar.) Apply this to (5.2).

REMARK 5.9. (i) In particular, if $f^{\prime} \in \operatorname{Lip}(\alpha, p)(1<p<\infty)$ then $\| f-$ $s(n ; f) \|_{\infty}=O\left(1 / n^{1+\alpha-1 / p}\right)$. Observe that the factor $\log n$, required when $p=\infty[22$, p. 120], does not appear here.

(ii) Corollary 5.8 extends in the expected way to higher derivatives and higher order moduli. 
5.10. Lebesgue's proof $[22$, p. 120$]$ of the Dini-Lipschitz Theorem provides also an estimate for the rate of convergence:

$$
\|f-s(n ; f)\|_{\infty}=O\left(\omega\left(n^{-1} ; \infty ; f\right) \log n\right) .
$$

For $g_{y}$ of Remark 5.5 that estimate is inferior by the factor $\log n$ to the estimate $O\left(n^{1-\gamma}\right)$ provided by (5.2). On the other hand, the latter estimate is identical with the naive $l_{1}$ inequality

$$
\left\|g_{\gamma}-s\left(n ; g_{\gamma}\right)\right\| \infty \leq 2 \sum_{n+1}^{\infty} \hat{g}_{\gamma}(k)=O\left(\sum_{n+1}^{\infty} k^{-\gamma}\right)
$$

(which can be seen to be optimal). In contrast, if $a \leq 1 / 2$ the sequence of coefficients of the Hardy-Littlewood function $\varphi_{a}(t) \doteq \sum_{1}^{\infty} \varepsilon_{n} n^{-a-1 / 2} e^{i n t}$ (where $\left\{\varepsilon_{n}\right\}_{1}^{\infty}$ is the Rudin-Shapiro sequence in which every $\varepsilon_{n}= \pm 1$ ) does not belong to $l_{1}$; so no similar estimate is feasible. If $a>1 / 2$ it yields only $O\left(n^{1 / 2-a}\right)$. The salient property of $\left\{\varepsilon_{n}\right\}$ is that $s_{\nu}(t) \doteq \sum_{1}^{\nu} \varepsilon_{n} e^{i n t}=O\left(\nu^{1 / 2}\right)$ uniformly with respect to $t \in[0,2 \pi] ;[20$, p. 129]. From this bound it is deduced in [22, p. 199] that $\omega\left(h ; \infty, \varphi_{a}\right)=O\left(h^{a}\right)$ for $0<a<1$. On the other hand, it is proved in [17] that the decreasing rearrangement $\hat{f}^{*}$ of the sequence $|\hat{f}|$ satisfies $\hat{f}^{*}(n)=O\left(n^{1 / p-1} \omega^{*}\left(n^{-1} ; p ; f\right)\right)$ for all $f \in L^{p}$ when $1 \leq p \leq 2$. Accordingly, $\omega\left(h ; p ; \varphi_{a}\right) \approx h^{a}$ if $0<a<1$ and $2 \leq p \leq$ $\infty$. Thus, for $0<a<1$ and $2 \leq p \leq \infty, \int_{0}^{1 / n} h^{-1-1 / p} \omega\left(h ; p ; \varphi_{a}\right) d h \approx$ $n^{-(a-1 / p)}$ if (and only if) $p>1 / a$. Thus we can conclude from (5.2) only that $\left\|\varphi_{a}-s\left(n ; \varphi_{a}\right)\right\|_{\infty}=O\left(n^{-b}\right)$ for all $b<a$. The Lebesgue estimate, $O\left(n^{-a} \log n\right)$, is better. (In fact, the "correct" estimate is $O\left(n^{-a}\right)$; for by partial summation (let $\delta=a+1 / 2$ )

$$
\begin{aligned}
\sum_{\nu=n}^{k} \varepsilon_{\nu} \nu^{-\delta} e^{i \nu t} & =\sum_{\nu=n}^{k}\left[\nu^{-\delta}-(\nu+1)^{-\delta}\right] s_{\nu}+(k+1)^{-\delta} s_{k}-n^{-\delta} s_{n-1} \\
& =\sum_{\nu=n}^{k} O\left(\nu^{-a-1}\right)+O\left(k^{-a}\right)+O\left(n^{-a}\right),
\end{aligned}
$$

with each $O$ uniform with respect to $t$. We obtain the asserted $O\left(n^{-a}\right)$ estimate for the tail by allowing $k \rightarrow \infty$. On the other hand, it is false that $E\left(n ; \infty ; \varphi_{a}\right)=o\left(n^{-a}\right)$; for by 3.3 such an estimate would imply that $\omega\left(n^{-1} ; \infty ; \varphi_{a}\right)=o\left(n^{-a}\right)$, which is false, as we have seen.)

5.11. The question arises whether an analog of (5.2) might be valid with $p=\infty$ whenever the integral on the right is convergent (which is the case, for instance, for the function $\varphi_{a}$, as we verified at the end of 5.10). Unfortunately, the answer is no. For $0<\alpha<1$ the function $f$ of $[22$, p. 315 , 
Example 10] belongs to $\operatorname{Lip} \alpha \equiv \Lambda(\alpha, \infty, \infty) \subset \Lambda(0, \infty, 1)$. Hence, with $p=\infty$ in the integral of (5.2), there results

$$
\|f-s(n ; f)\|_{\infty} \leq A \int_{0}^{1 / n} h^{\alpha-1} d h=O\left(n^{-\alpha}\right) .
$$

However, $f$ in fact satisfies $\|f-s(n ; f)\|_{\infty} \geq A n^{-\alpha} \log n$ for infinitely many values of $n$.

\section{Related results}

Our proof of Theorem 5.1 suggests a simple proof of the inclusion that we referred to in Section 3. Compare [7, Theorem 5].

Theorem 6.1. Suppose $1 \leq p \leq r \leq \infty, \alpha-1 / p \geq \beta-1 / r$, and $0<q \leq$ $\infty$. Then $\Lambda(\alpha, p, q) \subset \Lambda(\beta, r, q)$. In fact, there exists $A$ depending solely upon $\alpha, \beta, p, r, q$ such that, if $f \in \Lambda(\alpha, p, q)$, then $f \in L^{r}$ and

$$
\int_{0}^{1} \omega^{q}(h ; r ; f) h^{-1-\beta q} d h \leq A \int_{0}^{1} \omega^{q}(h ; p ; f) h^{-1-\alpha q} d h,(q<\infty),
$$

while

$$
\sup \left\{h^{-\beta} \omega(h ; r ; f): 0<h \leq 1\right\} \leq A \sup \left\{h^{-\alpha} \omega(h ; p ; f): 0<h \leq 1\right\},
$$

(corresponding to $q=\infty$ ).

Moreover, $\omega(h ; p ; f)=o\left(h^{\alpha}\right)$ implies $\omega(g ; r ; f)=o\left(h^{\beta}\right)$.

Proof. The case $p=r$ is trivial so we may exclude it. Also we assume $p>1$; see Remark 6.2. Suppose initially that $q<\infty$ and $f \in \Lambda(\alpha, p, q)$; thus, by Lemma 3.12, $f \in L^{p}$ and $\sum_{j=1}^{\infty} 2^{\alpha q j} E^{q}\left(2^{j} ; p ; f\right)<\infty$. Let $\sigma_{\nu} \doteq$ $s\left(2^{\nu+1} ; f\right)-s\left(2^{\nu} ; f\right)$. As in the proof of Theorem $5.1 f-s\left(2^{k}\right)=\sum_{\nu=k}^{\infty} \sigma_{\nu}$ converges in $L^{p}$. By Olivier's Theorem (3.10) $E(n ; p ; f)=O\left(n^{-\alpha}\right)$; so by 3.8 and the triangle inequality, $\left\|\sigma_{\nu}\right\|_{p} \leq A E\left(2^{\nu} ; p\right)=o\left(2^{-\alpha \nu}\right)$. By Remark 2.1, our hypothesis yields $\left\|\sigma_{\nu}\right\|_{r} \leq A 2^{\nu(1 / p-1 / r)}\left\|\sigma_{\nu}\right\|_{p}=o\left(2^{-\nu / \beta}\right)$. Therefore $\sum \sigma_{\nu}$ converges in $L^{r}$, and in particular, $f \in L^{r}$. Additionally,

$$
E\left(2^{k} ; r\right) \leq\left\|f-s\left(2^{k}\right)\right\|_{r} \leq \sum_{\nu=k}^{\infty} \mid \sigma_{\nu} \|_{r} \leq A \sum_{\nu=k}^{\infty} 2^{\nu(1 / p-1 / r)} E\left(2^{\nu} ; p\right) .
$$


Thus, for $2^{k} \leq n<2^{k+1}$, by 3.2 , condensation and 3.7 (we write $\gamma \doteq$ $1 / p-1 / r-1)$,

$$
\begin{aligned}
E(n ; r) \leq E\left(2^{k} ; r\right) & \leq A \sum_{m=2^{k}}^{\infty} m^{\gamma} \omega\left(m^{-1} ; p\right) \leq A \sum_{m=2^{k}}^{\infty}(2 m)^{\gamma} \omega\left((2 m)^{-1} ; p\right) \\
& \leq A \sum_{j=2^{k+1}}^{\infty} j^{\gamma} \omega\left(j^{-1} ; p\right) \leq A \sum_{j=n}^{\infty} j^{\gamma} \omega\left(j^{-1} ; p\right) .
\end{aligned}
$$

By this inequality and Lemma 3.11 (and therein the fact that $\beta+1 / p-1 / r \leq$ $\alpha)$ we conclude that

$$
\begin{aligned}
& \sum_{n=1}^{\infty} n^{\beta q-1} E^{q}(n ; r) \leq A \sum_{n=1}^{\infty} n^{\beta q-1}\left\{\sum_{j=n}^{\infty} j^{1 / p-1 / r-1} \omega\left(j^{-1} ; p\right)\right\}^{q} \\
& \quad \leq A \sum_{n=1}^{\infty} n^{-1+q(\beta+1 / p-1 / r)} \omega^{q}\left(n^{-1} ; p\right) \leq A \sum_{n=1}^{\infty} n^{-1+\alpha q} \omega^{q}\left(n^{-1} ; p\right) \\
& \quad \leq A \int_{1}^{\infty} t^{-1+\alpha q} \omega^{q}\left(t^{-1} ; p\right) d t=A \int_{0}^{1} h^{-1-\alpha q} \omega^{q}(h ; p) d h<\infty .
\end{aligned}
$$

With Lemma 3.12 this provides the asserted inequality between the integrals.

For the case $q=\infty$ observe that when $f \in \Lambda(\alpha, p, \infty)$, by $3.2, E\left(2^{\nu} ; p\right)$ $=O\left(2^{-\alpha \nu}\right)$; and, as above, this warrants $f-s\left(2^{k}\right)=\sum_{k}^{\infty} \sigma_{\nu}$ in $L^{r}$; so, as above, $E(n ; r) \leq A \sum_{n}^{\infty} j^{\gamma} \omega\left(j^{-1} ; p\right)$. Suppose

$$
M_{f} \doteq \sup \left\{h^{-\alpha} \omega(h ; p ; f): 0<h \leq 1\right\}<\infty .
$$

We obtain, therefore, that

$E(n ; r) \leq A \sum_{n}^{\infty} j^{\gamma} \omega\left(j^{-1} ; p\right) \leq A \sum_{n}^{\infty} j^{\gamma} M_{f} j^{-\alpha} \leq A M_{f} n^{1 / p-1 / r-\alpha} \leq A M_{f} n^{-\beta}$.

Thus, by Lemma 3.12 ,

$$
\sup \left\{h^{-\beta} \omega(h ; r): 0<h \leq 1\right\} \leq A \sup \left\{n^{\beta} E(n ; r): n \in \mathbb{Z}^{+}\right\} \leq A M_{f},
$$

as we asserted. The proof of the final implication is even simpler. Since $\gamma-\alpha \leq-\beta-1$ we have $E(\nu ; r) \leq A \sum_{\nu}^{\infty} o\left(j^{-1-\beta}\right)=o\left(\nu^{-\beta}\right)$. Hence by (3.3) (compare the proof of Lemma 3.12) $\omega\left(n^{-1} ; r\right) \leq A n^{-1} \sum_{1}^{n} o\left(\nu^{-\beta}\right)=o\left(\nu^{-\beta}\right)$ since $\beta<1$.

REMARK 6.2. When $p=1$ the proof of Theorem 6.1 fails (along with Riesz's inequality), but can be salvaged. When $q=1$ the proof is more tidy, not requiring Lemma 3.11. To illustrate these assertions we outline a proof of the fact that $\Lambda^{*}(1,1,1) \subset \Lambda\left(r^{-1}, r, 1\right)$ for $1<r<\infty$.

Replace the Fourier sums $s\left(2^{k} ; f\right)$ occurring in the proof of Theorem 6.1 by the de la Vallee-Poussin sums $\tau\left(2^{k}\right)$ of 3.9. Since the degree of $\tau\left(2^{k}\right)$ is 
$2^{k+1}-1$, terms derived from the application of Remark 2.1 in the proof will be altered, but the factors introduced thereby can be absorbed into discretionary constants. The $\tau$-analog, $f-\tau\left(2^{k}\right)=\sum_{k}^{\infty}\left(\tau\left(2^{\nu+1}\right)-\tau\left(2^{\nu}\right)\right)$, continues to hold in $L^{1}$, according to 3.9. Instead of using Lemma 3.11 to justify the final concatenate inequalities of the proof we reverse the order of summation to obtain

$$
\begin{aligned}
\sum_{k=0}^{\infty} 2^{k / r} E\left(2^{k} ; r\right) & \leq A \sum_{k=0}^{\infty} 2^{k / r} \sum_{\nu=k}^{\infty} 2^{\nu(1-1 / r)} E\left(2^{\nu} ; 1\right) \\
& =A \sum_{\nu=0}^{\infty} 2^{\nu(1-1 / r)} E\left(2^{\nu} ; 1\right) \sum_{k=0}^{\nu} 2^{k / r} \leq A \sum_{\nu=0}^{\infty} 2^{\nu} E\left(2^{\nu} ; 1\right) .
\end{aligned}
$$

Condensation and Lemma 3.12 lead to the asserted conclusion.

6.3. Functions in $\Lambda^{*}(1,1,1)$ neet not satisfy the Dini-Lipschitz condition. For suppose $\eta>0$ and $g(t)=g_{\eta}(t) \doteq(\log \delta /|t|)^{-\eta}$ on $[-\pi, \pi]$, where $\delta \doteq \pi e^{1+\eta}$ and $g$ is extended to $\mathbb{R}$ by $2 \pi$-periodicity. Since $g$ is increasing and concave on $[0, \pi]$, by $[14$, p. 44$] \omega(h ; \infty ; g)=g(h)$; so $g$ does not satisfy the Dini-Lipschitz condition if $\eta \leq 1$. On the other hand, we shall verify that $\omega^{*}(h ; 1 ; g) \leq A_{\eta} h(\log \delta / h)^{-\eta-1}$, so that $g \in \Lambda^{*}(1,1,1)$. To this end we write

$I \doteq \int_{0}^{\pi}|g(t+h)-2 g(t)+g(t-h)| d t=\int_{0}^{3 h}+\int_{3 h}^{\pi-2 h}+\int_{\pi-2 h}^{\pi} \doteq J+K+L$.

Evidently $g \in \operatorname{Lip}(1, \infty)$ on a neighborhood of $\pi$ (in fact, on $[\varepsilon, 2 \pi-\varepsilon]$ ); thus $L=\int_{\pi-2 h}^{\pi} O(h) d t=O\left(h^{2}\right)$. Further, from the mean value theorem for second differences and the fact that on $(0, \pi),\left|g^{\prime \prime}(t)\right| \leq \eta t^{-2}(\log \delta / t)^{-\eta-1}$ (which is increasing), we conclude that, for a suitable $\theta=\theta_{t} \in(-1,1)$,

$$
\begin{aligned}
K & \leq \int_{3 h}^{\pi-2 h} 4 h^{2}\left|g^{\prime \prime}(t+2 \theta h)\right| d t \\
& \leq 4 \eta h^{2} \int_{3 h}^{\pi-2 h}(t+2 \theta h)^{-2}(\log (\delta /(t+2 \theta h)))^{-\eta-1} d t \\
& \leq 4 \eta h^{2} \int_{3 h}^{\pi-2 h}(t-2 h)^{-2}(\log (\delta /(t-2 h)))^{-\eta-1} d t \\
& \leq 4 \eta h^{2} \int_{0}^{1 / h}(\log \delta x)^{-\eta-1} d x \sim 4 \eta h(\log \delta / h)^{-\eta-1}
\end{aligned}
$$

by paragraph 2 of Section 3 .

To estimate $J$ we use

$$
J \leq \int_{0}^{3 h}|g(t+h)-g(t)| d t+\int_{0}^{3 h}|g(t-h)-g(t)| d t \doteq J^{+}+J^{-} .
$$


It is evident from a sketch that

$$
J^{+} \leq \int_{0}^{h}[g(h)-g(t)] d t+3 h[g(4 h)-g(h)] \doteq J_{1}^{+}+J_{2}^{+} .
$$

Since $g^{\prime}$ decreases on $(0, \pi), J_{2}^{+} \leq 9 h^{2} g^{\prime}(h)=9 \eta h(\log \delta / h)^{-\eta-1}$, while

$$
\begin{aligned}
J_{1}^{+} & =h(\log \delta / h)^{-\eta}-\int_{0}^{h}(\log \delta / t)^{-\eta} d t \\
& =-\int_{1 / h}^{\infty} D_{u}\left[u^{-1}(\log \delta u)^{-\eta}\right] d u-\int_{1 / h}^{\infty} u^{-2}(\log \delta u)^{-\eta} d u \\
& =\eta \int_{1 / h}^{\infty} u^{-2}(\log \delta u)^{-\eta-1} d u \sim \eta h(\log \delta / h)^{-\eta-1}
\end{aligned}
$$

by paragraph 2 of Section 3. Thus $J^{+}=O\left(h(\log \delta / h)^{-\eta-1}\right)$. Turning to $J^{-}=\int_{0}^{h / 2}+\int_{h / 2}^{h}+\int_{h}^{3 h} \doteq J_{1}^{-}+J_{2}^{-}+J_{3}^{-}$, we see, by symmetry and set inclusion, that $J_{2}^{-}=J_{1}^{-}<J_{1}^{+}$. Since $J_{3}^{-}=\int_{0}^{2 h}[g(t+h)-g(t)] d t<$ $J^{+}, I \leq A_{\eta} h(\log \delta / h)^{-\eta-1}$, which is increasing; hence $\omega^{*}(h ; 1 ; g) \leq$ $A_{\eta} h(\log \delta / h)^{-\eta-1}$.

\section{Appendix}

We wish to show that $\|T\|_{\infty} \leq 3 n^{1 / p}\|T\|_{p}$, where $0<($ degree of $T) \leq n$ and $1 \leq p<\infty$. We suppose initially that $p \leq 2$ and note that, for the Dirichlet kernel, $\left\|D_{n}\right\|_{2}^{2}=2 n+1$. Hence, from the convolution inequality ([22, pp. 37, 38], [6, p. 54]), we obtain

$\|T\|_{\infty}^{2}=\left\|D_{n} * T\right\|_{\infty}^{2} \leq(2 n+1)(2 \pi)^{-1} \int_{0}^{2 \pi}|T|^{2-p}|T|^{p} \leq(2 n+1)\|T\|_{\infty}^{2-p}\|T\|_{p}^{p}$.

Hence $\|T\|_{\infty}^{p} \leq(2 n+1)\|T\|_{p}^{p}$, which verifies the asserted inequality if $1 \leq$ $p \leq 2$. A standard device extends the inequality to values of $p \in(2, \infty)$. For such $p$ the interval $[p, 2 p]$, of length $>2$, contains an even integer $2 m$. (Note that $2 \leq m \leq p$.) Apply what we have already proved, with the index $p / m \leq 2$, to the polynomial $T^{m}$. Thus

$$
\|T\|_{\infty}^{m}=\left\|T^{m}\right\|_{\infty} \leq 3(m n)^{m / p}\left\|T^{m}\right\|_{p / m} \leq 3 m n^{m / p}\|T\|_{p}^{m} .
$$

Hence $\|T\|_{\infty} \leq 3 n^{1 / p}\|T\|_{p}$ (since $(3 x)^{1 / x}$ decreases on $[1, \infty)$ ). 


\section{References}

[1] S. Aljančič and M. Tomič, 'Uber den Stetigkeitsmodul von Fourier-Reihen mit monotone Koeffzienten', Math. Z. 88 (1965), 274-284.

[2] J. Bergh and J. Löfström, Interpolation Spaces, (Springer-Verlag, 1976).

[3] O. V. Besov, 'Investigation of a family of function spaces in connection with theorems of imbedding and extension', (Russian), Trudy. Mat. Inst. Steklov. 60 (1961), 42-81. Amer. Math. Soc. Transl. (2) 40 (1964), 85-126.

[4] P. Butzer and H. Berens, Semi-groups of Operators and Approximation, (Springer-Verlag, 1967).

[5] P. Butzer and R. Nessel, Fourier Analysis and Approximation, Vol. I (Academic Press, 1971).

[6] R. Edwards, Fourier Series, a Modern Introduction, Vol. I, Second Edition (SpringerVerlag, 1979).

[7] J. F. Fournier and W. M. Self, 'Some sufficient conditions for uniform convergence of Fourier series', J. Math. Anal. App. 126 (1987), 355-374.

[8] A. M. Garsia, 'A remarkable inequality and the uniform convergence of Fourier series', Indiana Univ. Math. J. 25 (1976), 85-102.

[9] A. M. Garsia, 'Some combinatorial methods in real analysis', Proc. Internat. Congress Math. Helsinki (1978), 615-622.

[10] G. H. Hardy, J. E. Littlewood and G. Polya, Inequalities, (Cambridge University Press, 1952).

[11] G. H. Hardy and W. W. Rogosinski, Fourier Series, (Cambridge University Press, 1950).

[12] Y. Katznelson, An Introduction to Harmonic Analysis, (Wiley, 1968).

[13] K. Knopp, Theory and Application of Infinite Series, (Blackie, 1951).

[14] G. G. Lorentz, Approximation of Functions, (Holt, Rinehart, and Winston, 1966).

[15] I. P. Natanson, Constructive Function Theory, Vol. I (Ungar, 1964).

[16] C. C. Oehring, 'Asymptotics of singular numbers of smooth kernels via trigonometric transforms', J. Math. Anal. App. 145 (1990) 573-605.

[17] C. C. Oehring, 'Asymptotics of rearranged trigonometric and Walsh-Fourier coefficients of smooth functions', J. Math. Anal. App. (to appear).

[18] S. K. Pichorides, 'On the best values of the constants in the theorems of M. Riesz, Zygmund and Kolmogorov', Studia Math. 44 (1972) 165-179.

[19] M. K. Potapov and V. M. Fedorov, 'On Jackson theorems for the generalized modulus of smoothness', Studies in the Theory of Functions of Several Real Variables and the Approximation of Functions, Trudy Mat. Inst. Steklov. 172 (1985), 291-298. Translated in Proc. Steklov Inst. Mat. (1987), 315-323.

[20] T. J. Rivlin, The Chebyshev Polynomials, (Wiley, 1974).

[21] E. M. Stein, Singular Integrals and Differentiability Properties of Functions, (Princeton University Press, 1970).

[22] A. Zygmund, Trigonometric Series, Vol. I, (Cambridge University Press, 1959).

Virginia Polytechnic Institute \& State University

Blacksburg, Virginia 24061-0123

U.S.A. 